Supplemental Information for:

\title{
Mycobacterium tuberculosis exploits a heterohexameric enoyl-CoA hydratase retro-aldolase complex for cholesterol catabolism
}

Tianao Yuan ${ }^{\mathrm{a}, 1}$, Meng Yang ${ }^{\mathrm{b}, 1}$, Kalle Gehring ${ }^{\mathrm{b}}$, Nicole S. Sampson ${ }^{\mathrm{a},{ }^{*}}$

${ }^{a}$ Department of Chemistry, Stony Brook University, Stony Brook, NY 11794-3400

${ }^{b}$ Department of Biochemistry, McGill University, Montreal, Quebec, Canada

${ }^{1}$ Contributed equally to this work.

"Corresponding author:

$+1-631-632-7952$ (phone)

+1-631-632-5738 (fax)

nicole.sampson@stonybrook.edu 
Table S1. Expression constructs used in this work.

\begin{tabular}{|c|c|c|c|c|}
\hline Construct name & Genes & Restriction & Purified enzyme & Source/Reference \\
\hline$p E T 28 b$ & - & - & - & Novagen \\
\hline$p T i P Q C 1$ & - & - & - & Tomohiro Tamura \\
\hline pTipQC2 & - & - & - & Tomohiro Tamura \\
\hline pigr3 & $R v 3542 c, R v 3541 c, R v 3540 c$ & Ndel/HindIII & $\mathrm{N}-\mathrm{His}_{6}-\mathrm{ChsH} 2-\mathrm{ChsH} 1$ & 1 \\
\hline pChsH1-ChsH2 & Rv3541c, Rv3542c & Ndel/HindIII & $\mathrm{N}-\mathrm{His}_{6}-\mathrm{ChsH} 2-\mathrm{ChsH} 1$ & This work $^{a}$ \\
\hline pigr-3-RHA1 & $R v 3542 c, R v 3541 c, R v 3540 c$ & Ndel/HindIII & $\mathrm{N}-\mathrm{His}_{6}-\mathrm{ChsH} 2-\mathrm{ChsH} 1-\mathrm{Ltp} 2$ & This work ${ }^{\mathrm{b}}$ \\
\hline pigr-3_Ltp $2_{C 85 A}-R H A 1$ & $R v 3542 c, R v 3541 c, R v 3540 c$ & Ndel/HindIII & ChsH2-ChsH1-Ltp2-His 6 -C & This work ${ }^{c}$ \\
\hline pigr-3_Ltp2 ${ }_{\mathrm{C} 85 \mathrm{~A}, \mathrm{H} 344 \mathrm{~A}}-\mathrm{RHA1}$ & Rv3542c, Rv3541c, Rv3540c & Ndel/HindIII & ChsH2-ChsH1-Ltp2-His 6 -C & This work ${ }^{c}$ \\
\hline pigr-3_Ltp2 ${ }_{H 344 A}-R H A 1$ & Rv3542c, Rv3541c, Rv3540c & Ndel/HindIII & $\mathrm{N}-\mathrm{His}_{6}-\mathrm{ChsH} 2-\mathrm{ChsH} 1-\mathrm{Ltp} 2$ & This work $^{\mathrm{b}}$ \\
\hline
\end{tabular}

${ }^{a}$ Genes were cloned from H37Rv genomic DNA and ligated into pET28b with the indicated restriction sites to include an $\mathrm{N}$-terminal $\mathrm{His}_{6}$ fusion tag or both of the $\mathrm{N}$ - and $\mathrm{C}$-terminal $\mathrm{His}_{6}$ fusion tag. ${ }^{\mathrm{b}} \mathrm{Genes}$ were cloned from H37Rv genomic DNA and ligated into pTipQC1 with the indicated restriction sites to include an N-terminal $\mathrm{His}_{6}$ fusion tag. ${ }^{\mathrm{C}} \mathrm{Genes}$ were cloned from H37Rv genomic DNA and ligated into pTipQC2 with the indicated restriction sites to include a C-terminal $\mathrm{His}_{6}$ fusion tag. 


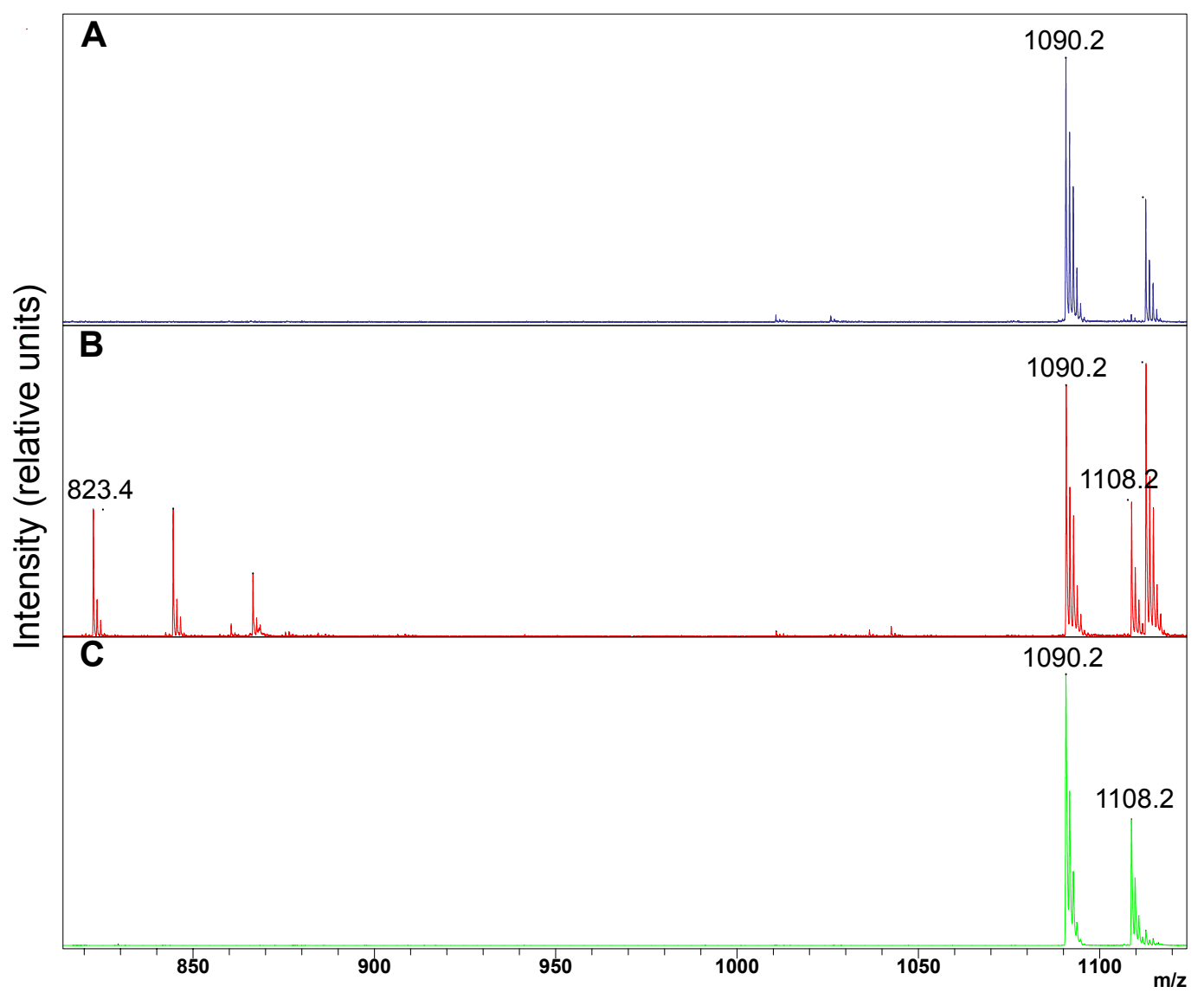

Figure S1. Propionyl-CoA formation from 3-OPDC-CoA requires full length ChsH1-ChsH2. (A) MALDI-TOF mass spectrum of 3-OPDC-CoA (1090.2 m/z, [M-H]'). (B) MALDI-TOF mass spectrum of 3-OPDC-CoA reaction mixture with the ChsH1ChsH2 preparation from the pigr-3 construct, 17-HOPC-CoA $\left(1108.2 \mathrm{~m} / \mathrm{z},[\mathrm{M}-\mathrm{H}]^{-}\right)$and propionyl-CoA (823.4 m/z, [M-H]'). (C) MALDI-TOF mass spectrum of 3-OPDC-CoA reaction mixture with the $\mathrm{ChsH} 1-\mathrm{ChsH} 2^{\mathrm{N}}$ preparation. ${ }^{1}$ 


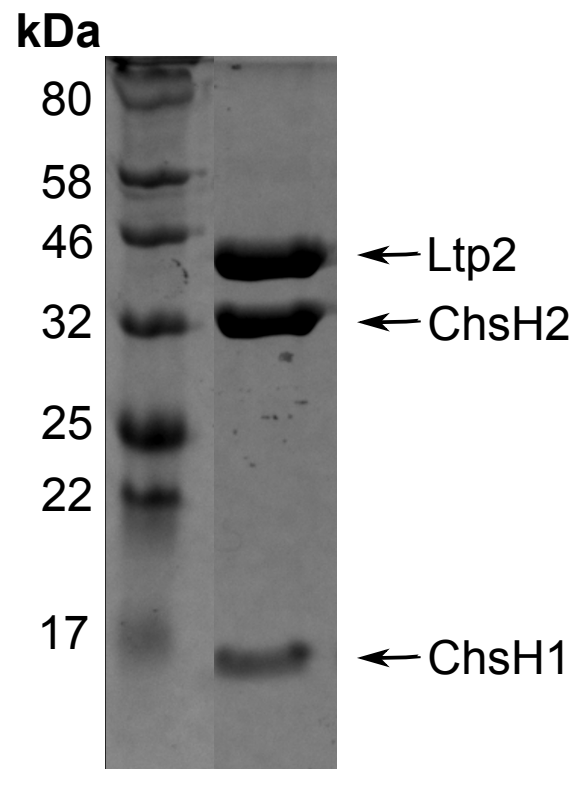

Figure S2. SDS-PAGE analysis of ChsH1-ChsH2-Ltp2. Construct pigr-3-RHA1 was used for the cistronic expression of ChsH1-ChsH2-Ltp2 (Uniprot IDs: I6XHI0-I6YGF816Y3T7) complex in Rhodococcus jostii RHA1. LB media cultures containing $30 \mu \mathrm{g} / \mathrm{mL}$ chloramphenicol were grown at $30^{\circ} \mathrm{C}$. Expression was induced at $O D_{600} \sim 0.6-0.8$ by the addition of $20 \mu \mathrm{g} / \mathrm{mL}$ thiostrepton, and cells were grown at $25^{\circ} \mathrm{C}$ for $24 \mathrm{~h}$ before harvesting, lysis, and IMAC nickel resin and SEC purification. 
A

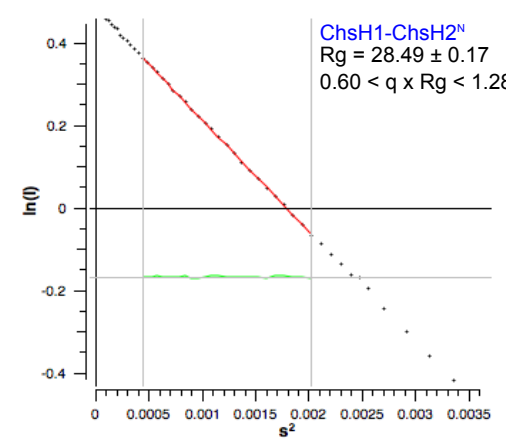

D

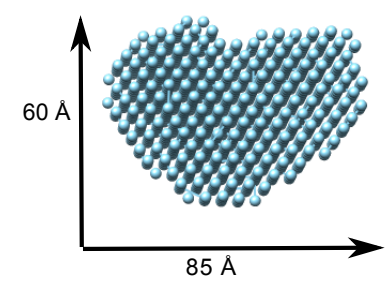

B

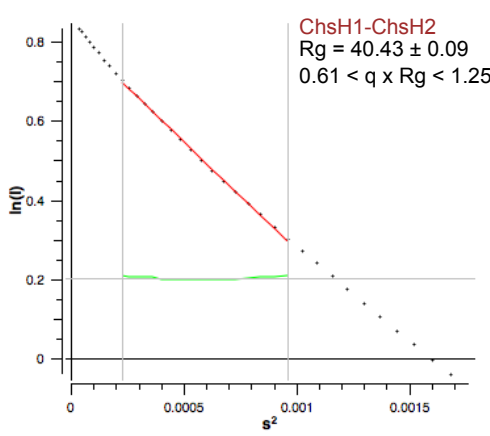

ChsH1-ChsH2

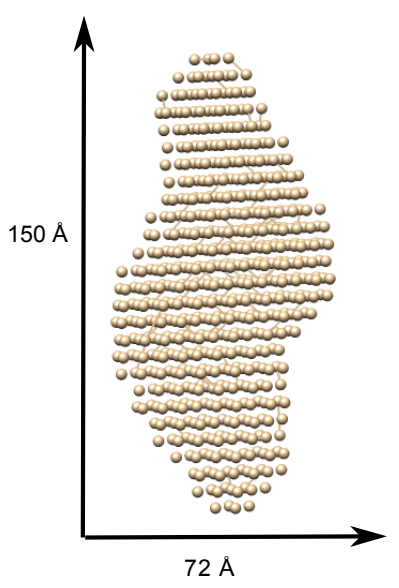

C

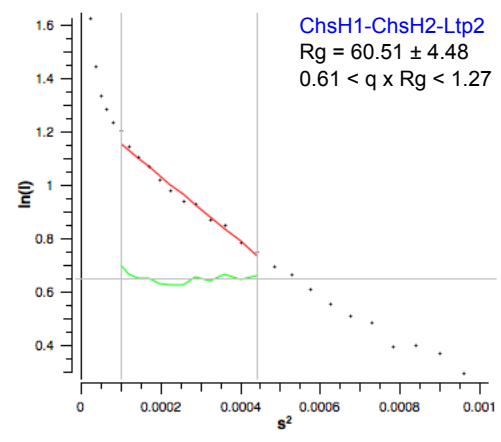

ChsH1-ChsH2-Ltp2

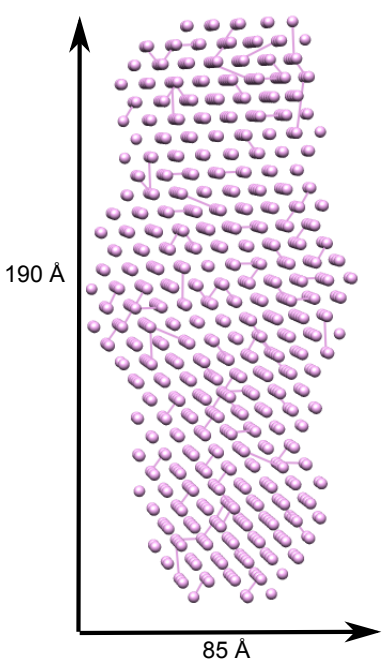

Figure S3. Guinier analysis of (A) ChsH1-ChsH2N, (B)ChsH1-ChsH2, and (C) ChsH1ChsH2-Ltp2. The Guinier analysis presents the linear region using the rule that $q \times \mathrm{R}_{\mathrm{g}}<$ 1.3. The fits are shown in red lines. The residuals of the fits are shown in green lines. (D) Bead models were generated from the X-ray scattering data of Chs $11-C h s H 2{ }^{\mathrm{N}}$, full length $\mathrm{ChsH} 1-\mathrm{ChsH} 2$, and $\mathrm{ChsH} 1-\mathrm{ChsH} 2-\mathrm{Ltp} 2$ complex. 
Table S2. SAXS parameters calculated from the Guinier plots

\begin{tabular}{cccc}
\hline & ChsH1-ChsH2 $^{\mathrm{N}}$ & ChsH1-ChsH2 & ChsH1-ChsH2-Ltp2 \\
\hline $\mathrm{R}_{\mathrm{g}}(\AA)$ & $28.49 \pm 0.17$ & $40.43 \pm 0.09$ & $60.51 \pm 4.48$ \\
$\mathrm{D}_{\max }(\AA)$ & 90.79 & 180.48 & 190.64 \\
Porod Voume & 54500 & 142000 & 309000 \\
\hline
\end{tabular}

A

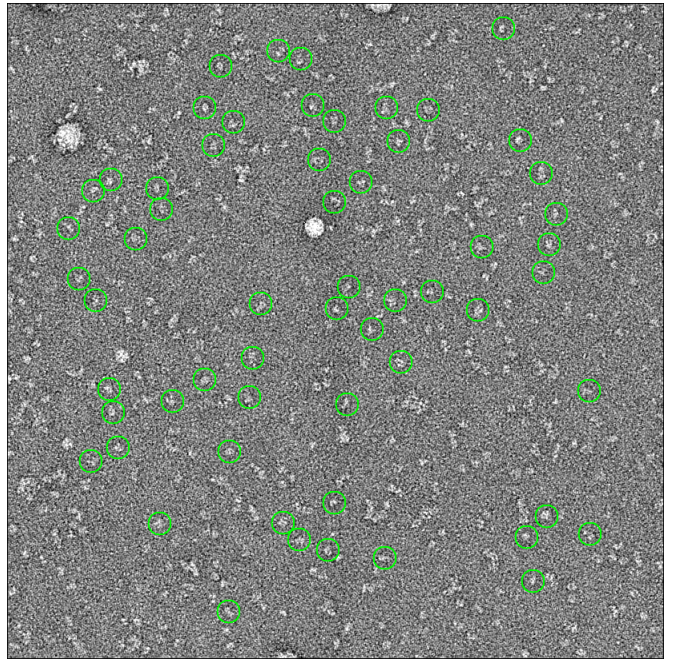

B

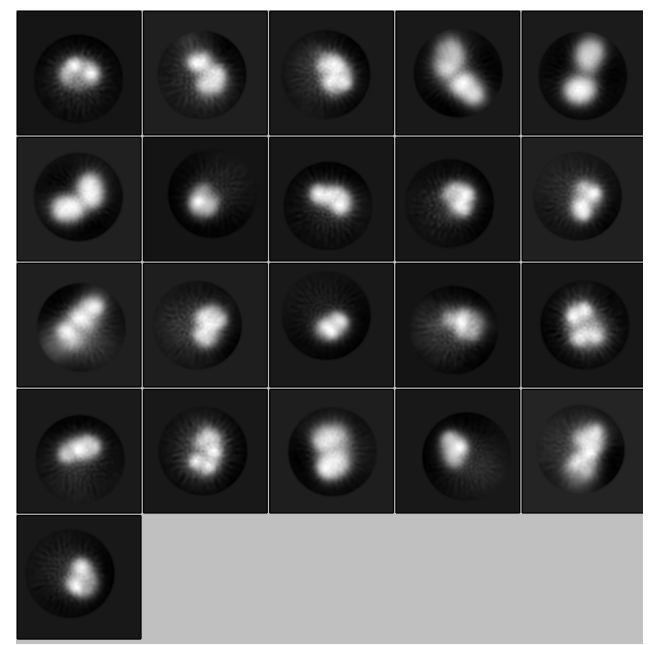

Figure S4. (A) Negative stain-EM image of ChsH1-ChsH2-Ltp2 complex. Particles boxed in green circles were selected. (B) 2D classes from particle alignments, generated in Relion3.0. 
2D classification and stack cleaning

116301 particles

$\downarrow$ Initial model (SGD algorithm)

$\downarrow$ 3D classification

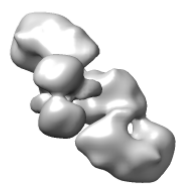

13711

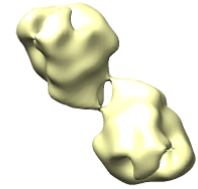

14351

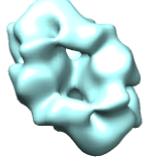

36804

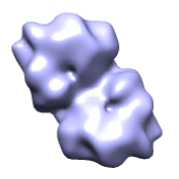

3689914536

3D refinement

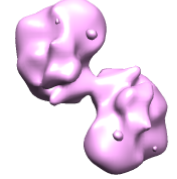

\section{$24 \AA$}

Figure S5. EM data processing scheme. 3D classification of the dataset results in five major classes. Each class has its corresponding particle number label underneath the structure. The best class was selected based on the similarity with the initial model and the number of particles. 3D refinement was performed on the selected class and particles. 


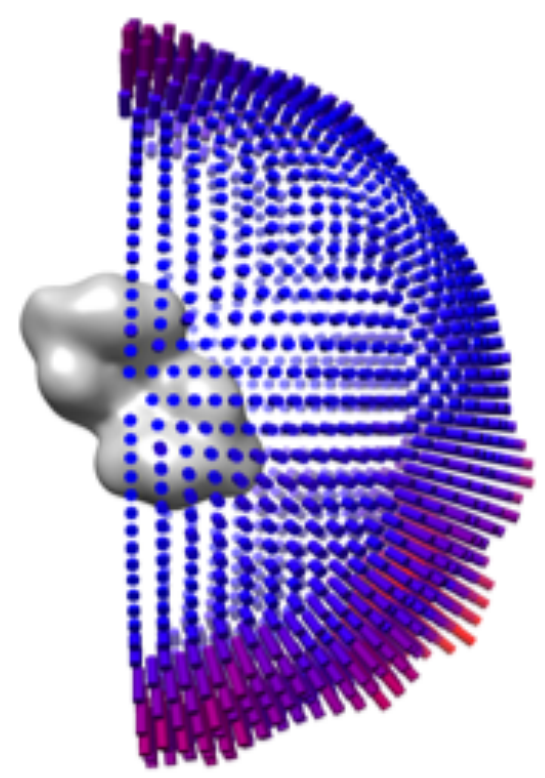

Figure S6. Angular distribution of the complex with a C2 symmetry showed the number of particles in each orientation. The plot shows that the complex adopts almost randomly distributed orientations on EM grid. 


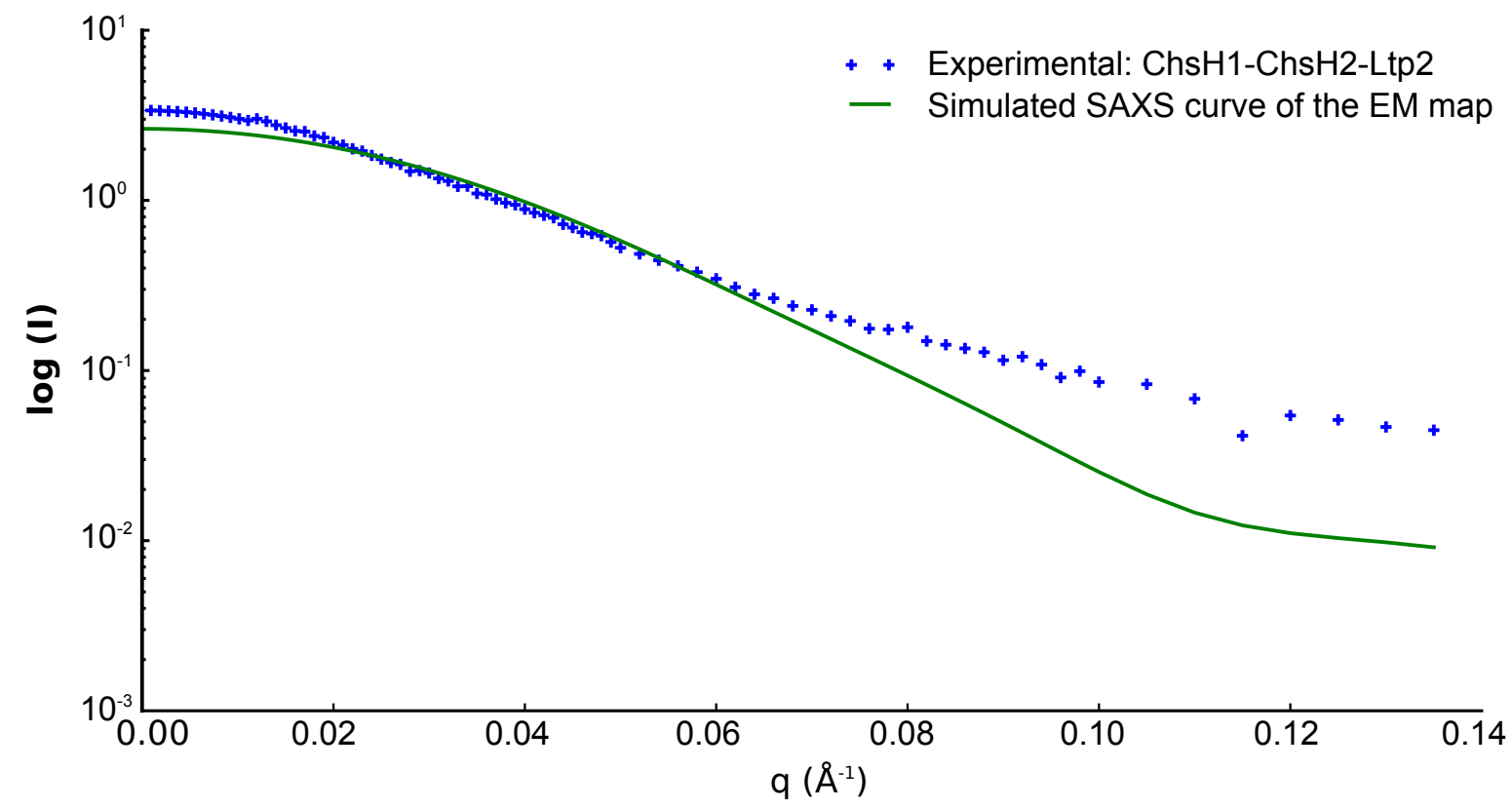

Figure S7. Experimental SAXS curve (blue) of ChsH1-ChsH2-Ltp2 compared with back-calculated scattering from the EM model. 
A

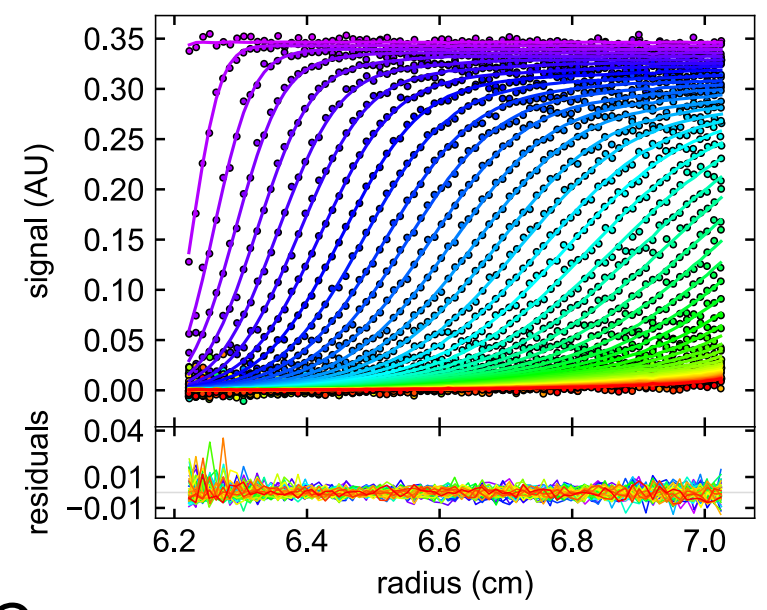

C

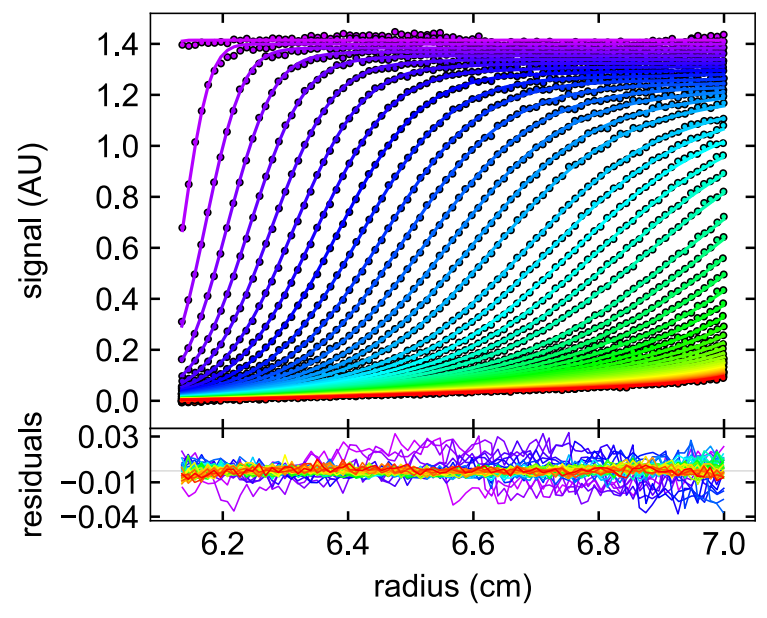

B

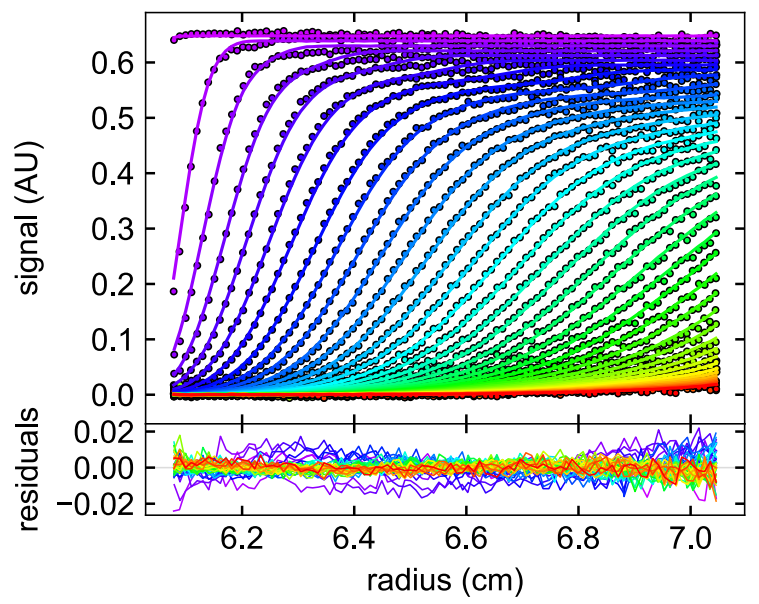

Figure S8. Representative sedimentation velocity AUC profile of ChsH1-ChsH2Ltp2 complex at different concentrations. (A) $0.425 \mathrm{mg} / \mathrm{ml} \mathrm{ChsH1-ChsH2-Ltp2.} \mathrm{(B)}$ $0.85 \mathrm{mg} / \mathrm{ml} \mathrm{ChsH1-ChsH2-Ltp2.} \mathrm{(C)} 1.7 \mathrm{mg} / \mathrm{ml} \mathrm{ChsH1-ChsH2-Ltp2.} \mathrm{The} \mathrm{absorbance} \mathrm{of}$ the sample at $280 \mathrm{~nm}$ and residuals are plotted against the radial position in the cell. One in every 10 scans is plotted. 
Table S3. SV-AUC sedimentation coefficients and estimated molecular weights

\begin{tabular}{cccccccc}
\hline & \multicolumn{6}{c}{ Sedimentation Coefficient (S) } & \multicolumn{3}{c}{ Estimated MW (kDa) } & RMSD \\
\hline $\begin{array}{c}\text { [Protein] } \\
\mathrm{mg} / \mathrm{ml}\end{array}$ & Peak 1 & Peak 2 & Peak 3 & Peak 1 & Peak 2 & Peak 3 & \\
\hline 0.425 & 4.364 & 7.553 & 12.701 & 73.8 & 159 & 350 & 0.003492 \\
\hline 0.850 & 4.738 & 7.674 & 12.689 & 83.7 & 171 & 347 & 0.004351 \\
\hline 1.700 & 4.903 & 7.806 & 12.802 & 84.9 & 181 & 354 & 0.005073 \\
\hline
\end{tabular}

A

B
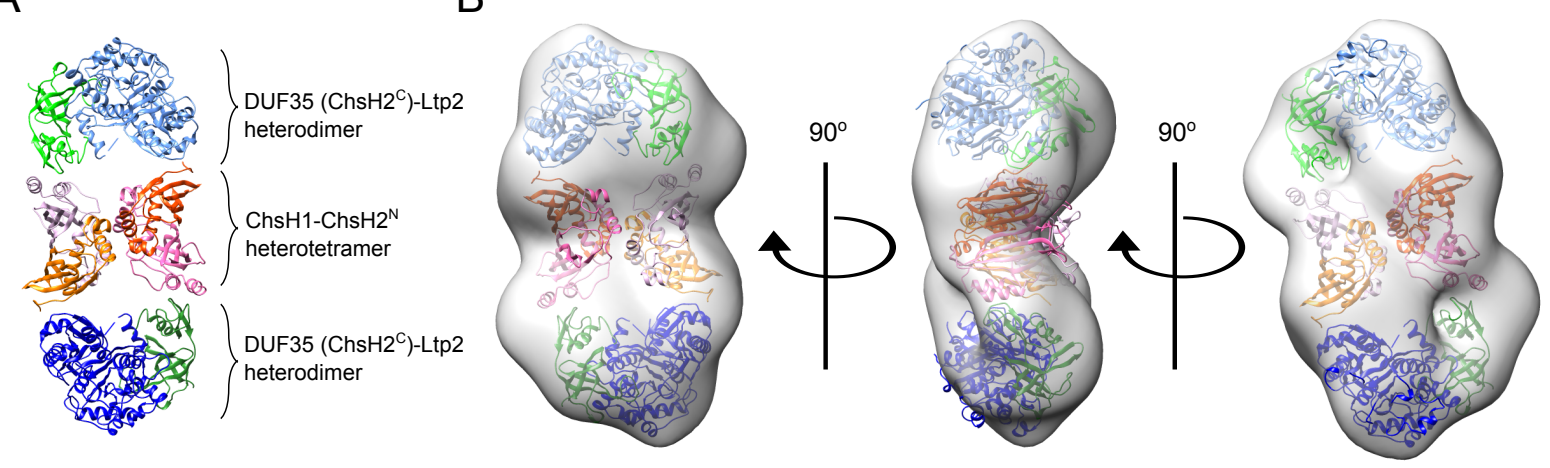

C

D
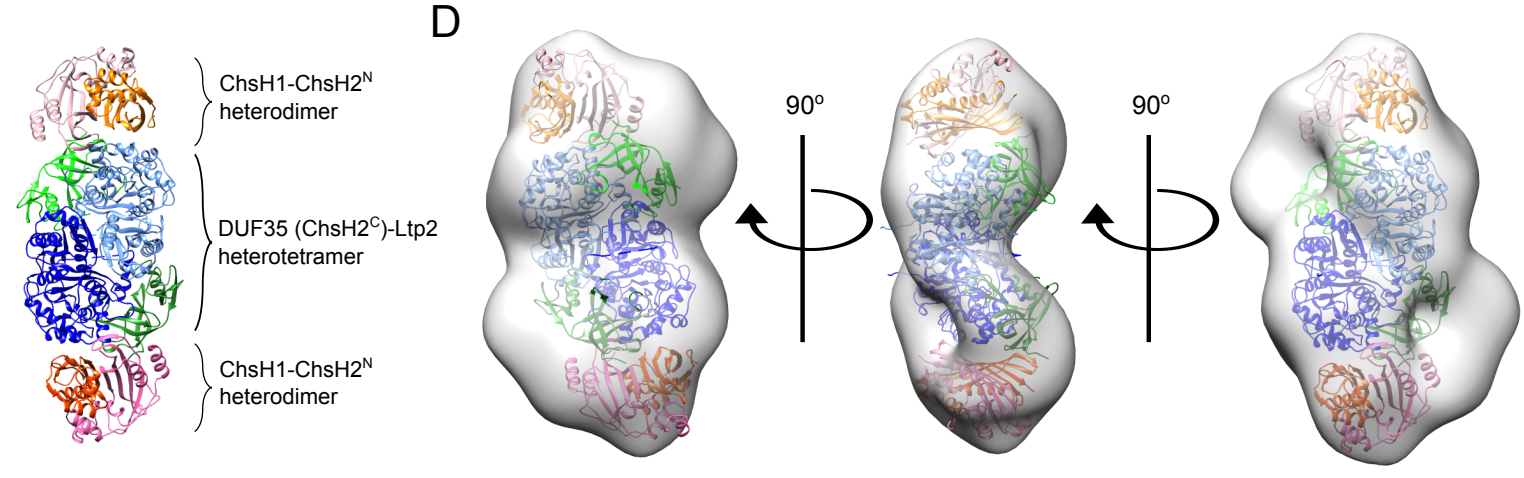

Figure S9. Rosetta protein-protein docking results. (A) Heterotetrameric $\mathrm{ChsH} 1-$ $\mathrm{ChsH} 2{ }^{\mathrm{N}}$ is in the middle and two copies of heterodimeric DUF35 $\left(\mathrm{ChsH}_{2}{ }^{\mathrm{C}}\right)-\mathrm{Ltp} 2$ are positioned at each end of the complex. (B) Different views of the model in (A) fitted into the EM map. (C) Heterotetrameric DUF35 $\left(\mathrm{ChsH}^{\mathrm{C}}\right)^{\mathrm{C}}$-Ltp2 ${ }_{\mathrm{T}}$. curvata is in the middle and two copies of heterodimeric $\mathrm{ChsH} 1-\mathrm{ChsH} 2^{\mathrm{N}}$ are on the two ends. (D) Different views of the model in (C) fitted into the EM map. $\mathrm{ChsH} 1$ chains are colored in different shades of orange, $\mathrm{ChsH} 2^{\mathrm{N}}$ chains are colored in different shades of pink, DUF35 T. curvata $\left(\mathrm{ChsH}^{\mathrm{C}}\right)$ chains are colored in different chains of green, and Ltp2 ${ }_{T}$. curvata chains are colored in different shades of blue. 


\section{Reference:}

[1] Yang, M., Guja, K. E., Thomas, S. T., Garcia-Diaz, M., and Sampson, N. S. (2014) A distinct MaoC-like enoyl-CoA hydratase architecture mediates cholesterol catabolism in Mycobacterium tuberculosis, ACS Chem Biol 9, 2632-2645. 